03.1;03.4

\title{
Влияние поверхностного натяжения на разрушение макрообъема жидкости при его свободном падении
}

\author{
(ㄱ В.А. Архипов, С.А. Басалаев , Н.Н. Золоторёв, К.Г. Перфильева, А.С. Усанина \\ Национальный исследовательский Томский государственный университет, Томск, Россия \\ 『 E-mail: tarm@niipmm.tsu.ru
}

Поступило в Редакцию 9 июля 2019г.

В окончательной редакции 29 октября 2019 г.

Принято к публикации 1 ноября 2019г.

\begin{abstract}
Представлены методика и результаты экспериментального исследования влияния коэффициента поверхностного натяжения и начального объема водяного ядра на динамику его разрушения при свободном падении в воздухе с ненулевой начальной скоростью. Показано, что при двукратном снижении коэффициента поверхностного натяжения жидкости расстояние, на котором происходит полное разрушение водяного ядра с образованием облака капель, сокращается на $30 \%$, а зависимость расстояния полного разрушения от начального объема ядра имеет минимум.
\end{abstract}

Ключевые слова: макрообъем жидкости, свободное падение, расстояние полного разрушения, коэффициент поверхностного натяжения, начальный объем.

DOI: 10.21883/PJTF.2020.03.48989.17970

Процессы разрушения достаточно больших массивов жидкости с образованием облака жидкокапельного аэрозоля при свободном падении в воздушной среде реализуются при сбросе гарантийного запаса жидкого ракетного топлива отделяющихся ступеней ракет-носителей и при аварийном сбросе авиационного топлива $[1,2]$. Одним из распространенных способов тушения крупных пожаров, особенно в труднодоступных районах, является сброс хладагента (как правило, воды) в очаг пожара с борта самолета или вертолета с помощью специальных шлюзовых систем [3]. Для повышения эффективности технологии авиационного пожаротушения необходимо моделирование процесса разрушения макрообъема жидкости, сбрасываемого с заданной скоростью из водосливного устройства летательного аппарата, с образованием первичного облака полидисперсных капель.

Получение экспериментальных количественных данных по расстоянию полного разрушения массива жидкости необходимо как для использования в конкретных задачах, так и для оценки адекватности разрабатываемых математических моделей процесса [4]. Результаты экспериментальных исследований качественной картины динамики разрушения макрообъема жидкости при свободном падении в воздухе с нулевой начальной скоростью приведены в [5-9].

В настоящей работе представлены методика и результаты экспериментального исследования влияния коэффициента поверхностного натяжения жидкости и начального объема водяного ядра на динамику его разрушения при свободном падении в воздухе с ненулевой начальной скоростью.

Экспериментальная установка (рис. 1, $a$ ) состоит из устройства 1 для прокалывания стенки заполненной жидкостью эластичной оболочки 2, установленного на вертикальной штанге 3, и системы визуализации процесса разрушения сферического макрообъема жидкости 4 с помощью двухракурсной съемки видеокамерами 5 типа Citius C100 с темпом съемки 50-1000 кадров в секунду [10]. Устройство для равномерного прокалывания стенки оболочки (рис. $1, b$ ) состоит из кольца 6 , по окружности которого равномерно расположены три заостренные стальные иглы 7 диаметром $0.45 \mathrm{~mm}$, и цилиндрической направляющей втулки 8 , расположенной в верхней части кольца 6. Внутренняя стенка цилиндрической втулки 8 покрыта тонким слоем 9 антифрикционной смазки.

При проведении экспериментов формирование сферического макрообъема жидкости осуществлялось следующим образом. Тонкая эластичная оболочка (толщиной менее $0.5 \mathrm{~mm}$ ) наполнялась жидкостью, при этом формировалась сферическая частица с заданным начальным объемом $V$. Наполненная жидкостью оболочка 2 через цилиндрическую втулку 8 поступала в кольцо 6 (рис. $1, b)$. После разрыва оболочки формировался сферический макрообъем жидкости (рис. 1,c), движущейся с заданной начальной скоростью. Эволюция формы водяного ядра (рис. $1, d$ ) в процессе свободного падения на различных расстояниях фиксировалась видеосъемкой.

Представленные методика и экспериментальная установка для ее реализации [10] в отличие от используемых в работах [5-9] позволяют исследовать эволюцию макрообъема жидкости при падении с заданной ненулевой начальной скоростью и обеспечивают практически сферическую форму начального ядра жидкости. Использование кольца с равномерно расположенными по его окружности заостренными иглами, направленными радиально в сторону оси симметрии кольца, обеспечивает симметричное прокалывание стенок оболочки. Это 


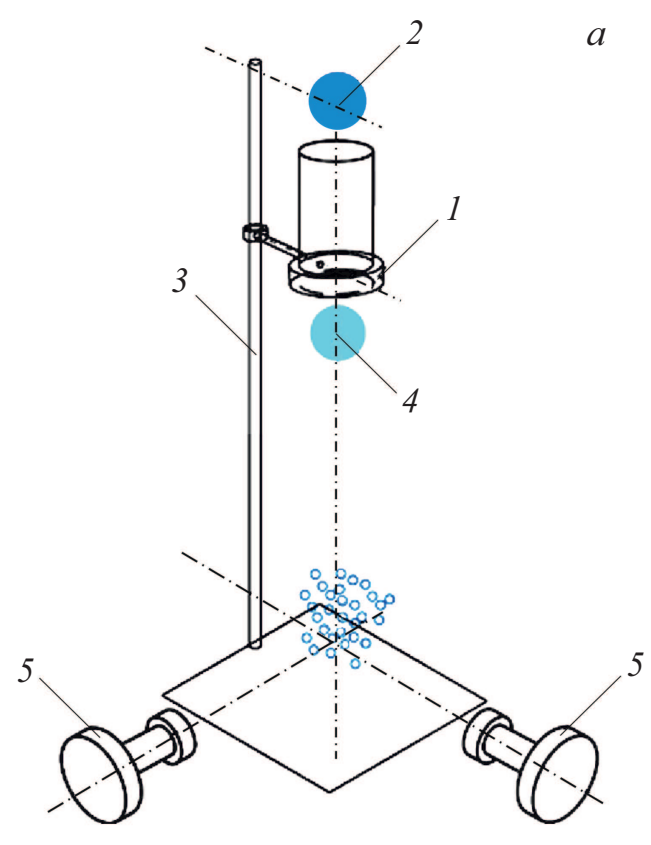

C

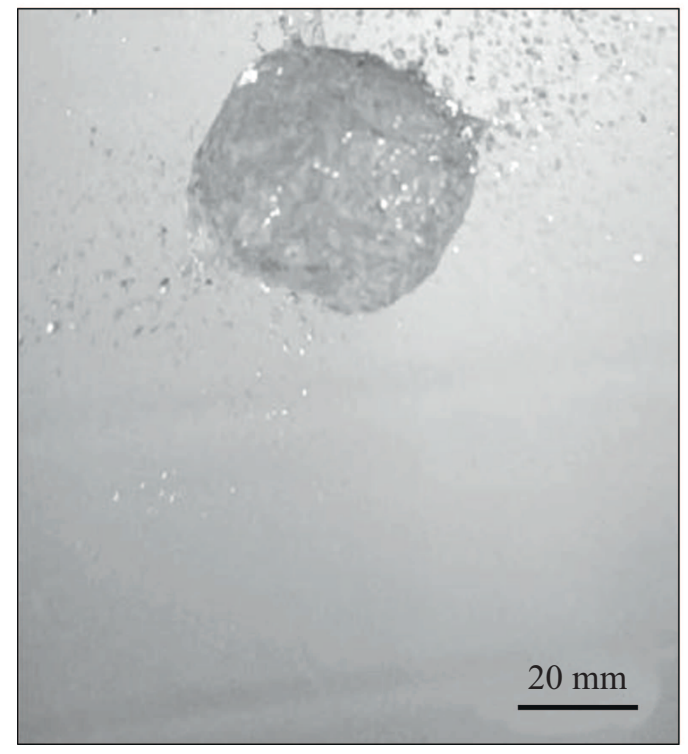

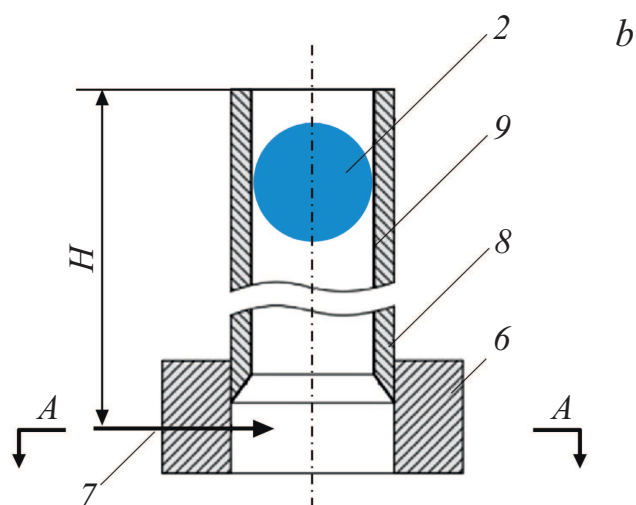

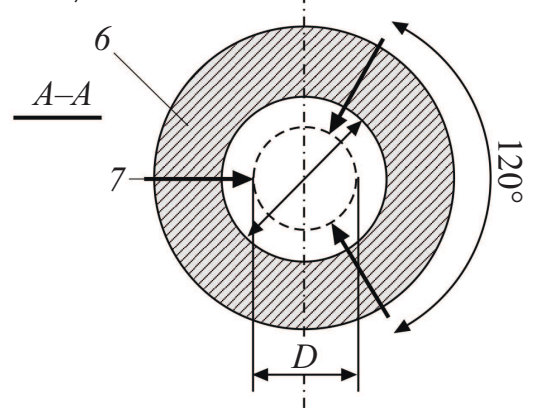

$d$

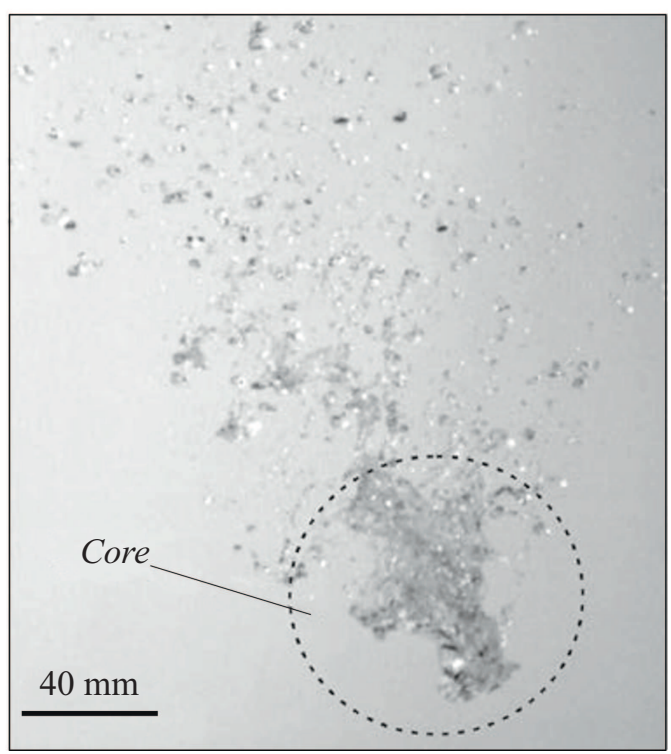

Рис. 1. Схема экспериментальной установки для исследования динамики разрушения сферического макрообъема жидкости $(a)$, схема устройства для прокалывания стенки оболочки $(b)$ и видеокадры, полученные для начального жидкостного ядра $(V=60 \mathrm{ml})$ $(c)$ и в процессе его свободного падения $(x=300 \mathrm{~cm})(d)$. Пояснения приведены в тексте.

исключает возможность наблюдаемого в работах $[6-8]$ неконтролируемого нерегулярного движения образовавшегося водяного ядра за счет дисбаланса сил, действующих со стороны фрагментов оболочки на макрообъем жидкости, или реактивной силы, создаваемой струей жидкости при прокалывании одиночного отверстия.

В режиме свободного падения исключается начальная деформация оболочки, наблюдаемая в случае ее подвешивания на стержне или трубке [5-9]. Соотношения для внутренних диаметров кольца и втулки, высоты выступающих на внутренней поверхности кольца игл получены по результатам проведенных экспериментов. Эти соотношения обеспечивают начальную форму макрообъема жидкости, наиболее близкую к сферической (рис. 1,c).

Соотношение для высоты цилиндрической направляющей втулки $H$ определялось исходя из условия обеспечения заданной начальной скорости жидкостного ядра в режиме свободного падения наполненной жидкостью оболочки внутри втулки. При движении наполненной 


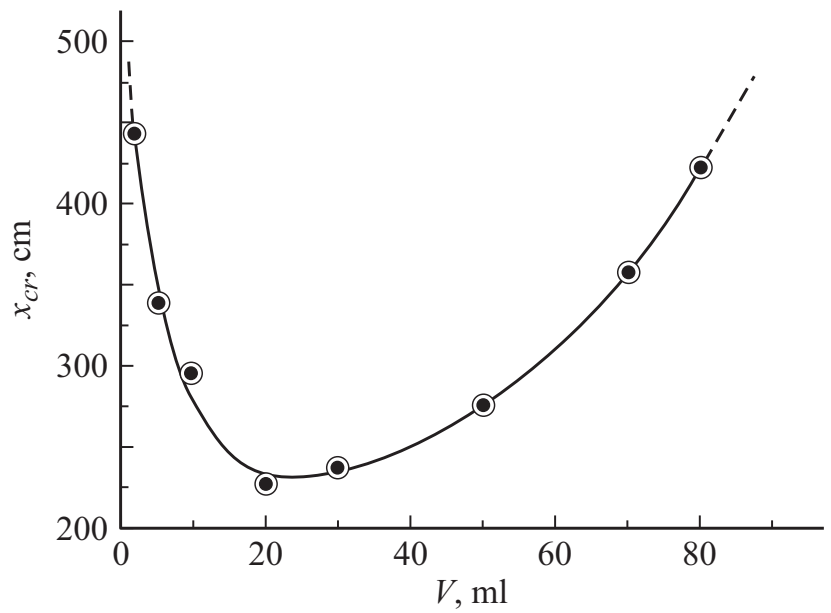

Рис. 2. Зависимость расстояния $x_{c r}$, пройденного жидкостным ядром до его полного разрушения, от начального объема.

жидкостью оболочки на нее действуют силы тяжести $F_{g}$ и аэродинамического сопротивления $F_{c}$

$$
F_{g}=\rho_{l} \frac{\pi D^{3}}{6} g, \quad F_{c}=C_{x} \frac{\pi D^{2}}{4} \frac{\rho_{g} u^{2}}{2},
$$

а начальная скорость жидкостного ядра определяется соотношением

$$
u=\sqrt{2 g H} .
$$

Здесь $\rho_{l}, \rho_{g}-$ плотности жидкости и воздуха, $D-$ диаметр жидкостного ядра, $g-$ ускорение свободного падения, $C_{x}=0.44-$ коэффициент сопротивления.

Из (1), (2) следует соотношение для высоты цилиндрической направляющей втулки

$$
H \leqslant 0.015 \frac{\rho_{l}}{\rho_{g}} D
$$

В качестве исследуемой жидкости использовалась дистиллированная вода. Для снижения коэффициента поверхностного натяжения в жидкость добавлялся додецилсульфат натрия в качестве поверхностно-активного вещества (ПАВ). Температура окружающей среды и раствора вода + ПАВ составляла $20^{\circ} \mathrm{C}$. Начальный объем ядра варьировался в диапазоне $V=2-90 \mathrm{ml}$, коэффициент поверхностного натяжения жидкости - в диапазоне $\sigma=35-72 \mathrm{mN} / \mathrm{m}$. Эволюция жидкостного ядра при его начальной скорости $u=1.95 \mathrm{~m} / \mathrm{s}$ фиксировалась на расстояниях $x=0-7.5 \mathrm{~m}$.

Анализ экспериментальных данных показал, что процесс эволюции макрообъема жидкости можно разделить на следующие стадии: 1) движение сферического ядра жидкости; 2) постепенная трансформация ядра с образованием дискообразной конфигурации; 3) образование пузырей (,парашютов“); 4) разрушение пузырей с образованием жидкостных „цепочек“; 5) формирование жидкокапельного аэрозольного облака. Качественная картина различных стадий процесса для дистиллированной воды согласуется с результатами [5-9].
Для оценки достоверности результатов исследования проведено сравнение зависимости площади поперечного миделева сечения жидкостного ядра, отнесенной к его начальному сечению $\left(S / S_{0}\right)$, от пройденного расстояния $x$ до начала распада ядра с данными $[7,8]$. Обработка видеокадров движения макрообъема дистиллированной воды $(V=50 \mathrm{ml})$ показала, что на начальном участке траектории движения макрообъема жидкости $x \leqslant 2 \mathrm{~m}$ (начало распада жидкостного ядра) полученные значения $S / S_{0}$ с учетом разброса экспериментальных данных практически совпадают с результатами $[7,8]$. Отметим, что в работе [8] при $x>1-1.5 \mathrm{~m}$ наблюдалось существенное различие площади миделева сечения ядра, измеренного в разных ракурсах (съемка сверху и сбоку), что свидетельствует о явно выраженной несимметричности формы жидкостного ядра.

Результаты исследования влияния начального объема ядра жидкости показали, что зависимость расстояния $x_{c r}$, на котором происходит полное его разрушение, носит немонотонный характер (рис. 2). При малых размерах жидкостного ядра $(V<20 \mathrm{ml})$ силы поверхностного натяжения превалируют над силами динамического напора (числа Вебера меньше критического значения), и расстояние, на котором происходит полное его разрушение, $x_{c r}>220 \mathrm{~cm}$. При увеличении размеров ядра величина $x_{c r}$ достигает минимального значения. С дальнейшим увеличением размеров жидкостного ядра величина $x_{c r}$ монотонно возрастает. Это связано с тем, что после стадии разрушения пузырей с образованием жидкостных цепочек (рис. $1, d)$ жидкостное ядро полностью не разрушается, а постепенно уменьшается в размерах.

На рис. 3 приведены экспериментальные данные по влиянию коэффициента поверхностного натяжения жидкости $\sigma$ на расстояние $x_{c r}$ для жидкостного ядра с начальным объемом 20 и $40 \mathrm{ml}$. Из приведенных зависимостей следует, что коэффициент поверхностного

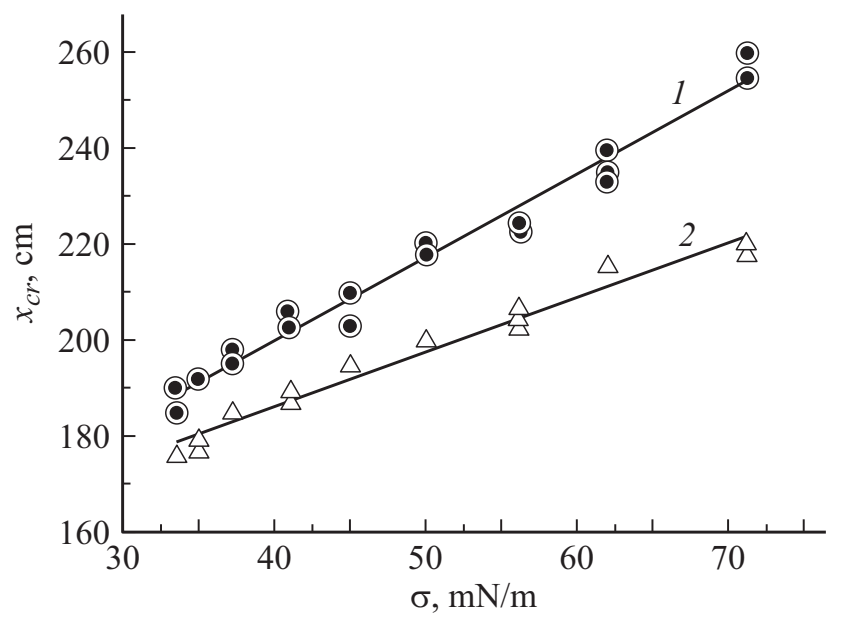

Рис. 3. Зависимость расстояния $x_{c r}$, пройденного макрообъемом жидкости до его полного разрушения, от коэффициента поверхностного натяжения жидкости $\sigma . V=40$ (1) и $20 \mathrm{ml}(2)$. 
натяжения жидкости существенно влияет на динамику разрушения макрообъема жидкости. При введении ПАВ в дистиллированную воду с уменьшением коэффициента поверхностного натяжения от 72 до $35 \mathrm{mN} / \mathrm{m}$ расстояние $x_{c r}$, на котором происходит разрушение макрообъема жидкости, сокращается от 255 до $185 \mathrm{~cm}$. Этот эффект необходимо учитывать при моделировании процесса сброса хладагента.

Таким образом, предлагаемый метод исследования обеспечивает более адекватное моделирование процесса сброса макрообъема жидкости из шлюзовых систем авиационного судна за счет учета ненулевой начальной скорости падения жидкостного ядра. Экспериментально обнаружено, что одним из наиболее значимых параметров процесса эволюции жидкостного ядра при свободном падении является коэффициент поверхностного натяжения жидкости. Введение небольшого количества ПАВ в состав хладагента позволяет существенно сократить расстояние, на котором образуется полидисперсное аэрозольное облако.

\section{Финансирование работы}

Исследование выполнено за счет гранта Российского научного фонда (проект № 15-19-10014).

\section{Конфликт интересов}

Авторы заявляют, что у них нет конфликта интересов.

\section{Список литературы}

[1] Архипов В.А., Жарова И.К., Козлов Е.А., Ткаченко А.С. // Оптика атмосферы и океана. 2015. Т. 28. № 1. С. 89-93.

[2] Arkhipov V.A., Kozlov E.A., Titov S.S., Tkachenko A.S., Usanina A.S., Zharova I.K. // Arab. J. Geosci. 2016. V. 9. P. $114(1-10)$.

[3] Асовский В.П. // Науч. вестник МГТУ ГА. Сер. Аэромеханика и прочность. 2009. № 138. С. 142-149.

[4] Кудров М.А. // Науч. вестник МГТУ ГА. Сер. Аэромеханика и прочность. 2010. № 151. С. 163-168.

[5] Мешков Е.Е., Орешков В.О., Янбаев Г.М. // Письма в ЖТФ. 2011. Т. 37. В. 15. С. 79-86.

[6] Волков Р.С., Забелин М.В., Кузнецов Г.В., Стрижак П.А. // Письма в ЖТФ. 2016. Т. 42. В. 5. С. 65-73.

[7] Накоряков В.Е., Кузнецов Г.В., Стрижак П.А. // ДАН. 2017. T. 475. № 2. С. 145-149.

[8] Накоряков В.Е., Кузнецов Г.В., Стрижак П.А. // ДАН. 2016. T. 467. № 5. C. 537-542.

[9] Reyssat É., Chevy F., Biance A.L., Petitjean L., Quéré D. // Europhys. Lett. 2007. V. 80. N 3. P. 34005.

[10] Архипов В.А., Басалаев С.А., Булавко А.Н., Золоторев Н.Н., Перфильева К.Г., Поленчук С.Н. Установка для исследования динамики разрушения сферического макрообъема жидкости при свободном падении в воздухе. Заявка № 2019116949 на патент РФ. МПК B01L 99/100. Заявл. 15.06.2019. 